\title{
Uber den Begriff des Unendlichen und der intelligibeln Ausdehnung bei Malebranche und die Beziehung des letzteren zum Kantischen Raumbegriff.
}

Von Dr. Artur Buchenau.

Man pflegt in der Geschichte der Philosophie Malebranche als getreuen Schüler Descartes hinzustellen, von dem er ja in der Tat den entscheidenden Anstoss zur Bildung seiner Lehre erhalten hat, und fasst demgemäss seine Philosophie ebenso wie die seines Meisters als Rationalismus. Dass bei aller Übereinstimmung in den grossen Zügen wie auch in vielen Einzelheiten dennoch grosse Verschiedenheiten in der Lehre beider bestehen, kann hier des genaueren nicht gezeigt werden, es sei nur darauf hingewiesen wie das auch bereits Ernst Cassirer im ersten Bande seines Werkes über das Erkenntnisproblem in der Philosophie und Wissenschaft der neueren Zeit betont hat - dass Malebranches Idealismus stark psychologisch gefärbt ist. Hierin liegt ja allerdings auf der einen Seite ein Abbiegen von dem geraden Wege der Erkenntnistheorie, der von Descartes und Galilei über Leibniz zu Kant führt, andererseits . aber doch eine wertvolle Ergänzung der Kartesischen Ideenlehre.

Auf dem Boden der Ideenlehre also hat man in der Hauptsache die Selbständigkeit Malebranches seinem grossen Lehrer gegenüber zu suchen, und es sind da besonders die beiden Begriffe des Unendlichen und der intelligibeln Ausdehnung, die einen deutlichen Fortschritt über Descartes hinaus bezeugen, ja, es lässt sich zeigen, dass der letztere Begriff eine geradezu auffallende Ähnlichkeit mit der Art und Weise aufweist, wie Kant in der Vernunftkritik den Raum als „reine Anschaunng“ charakterisiert.

Fasst man, wie erwähnt, die Philosophie Malebranches als Rationalismus, so ist damit noch nicht viel gewonnen; denn dieser Begriff ist kein eindeutiger. Mit inm lassen sich die widerstreitendsten Tendenzen vereinigen, so tritt er bei Augustin mit 
dem Spiritualismus, bei Hobbes mit dem Materialismus verbunden auf. Der Begriff der "ratio“ allein bietet eben keine Gewähr dafür, dass man nicht zu der Behauptung einer an und für sich Bestand habenden, sei es immateriellen, geistigen oder materiellen "Welt" fortschreitet. Auf diesem Standpunkte bleibt, wie man meint, der Veruunft und ihren Gesetzen ihr volles Recht, aber letztlich dient sie doch nur dazu, die als "gegeben“ vorausgesetzte Wirklichkeit zu beweisen. Nur die Vorstellung ist ja wahr, die mit dem vorgestellten Gegenstande völlig übereinstimmt. Und indem man nun vergeblich nach dem Kriterium für diese Übereinstimmung sucht, führt der dogmatische Rationalismus zum Skeptizismus, d. h. aber zur Gefährdung der Wissenschaft. Diesen Weg konnte Malebranche, der Schüler Descartes, der von dem Meister gelernt hatte, seine Philosophie in unmittelbare Beziehung zu der Wissenschaft zu setzen, diesen Abweg konnte er nicht betreten. So musste er dazu geführt werden, die Vernunft anders aufzufassen, vor allem aber die Annahme einer solchen, für sich bestehenden Wirklichkeit aufzugeben. So verstand er im alten Platonischen Sinne die Vernunft als das Vermögen der Prinzipien, als das Vermögen der Ideen. Von diesen, so nahm er mit Descartes an, muss seinen Ausgang nehmen, wer über die "Dinge“ etwas ausmachen will, denn fundamentaler und der Erkenntnis nach „früher" sind die Gesetze des Intellekts, der Vernunft, als die der Dinge, welch letztere sich in ihrer unübersehbaren Mannigfaltigkeit allein bewältigen lassen durch die einfachen, ganz "uns" angehörenden „Ideen“, d. h. Gesetzlichkeiten unseres Geistes.

So wird bei Malebranche der Rationalismus zum Idealismus. Auch mit dieser Charakterisierung aber sind noch keineswegs alle Schwierigkeiten beseitigt. Von den Ideen soll ausgegangen, auf sie soll alles zurückgeführt werden - wird damit nun nicht alles „subjektiv“? Denn was sind die Ideen anders als unser subjektives Denken! Und sollte wirklich unser Denken die Norm der Wahrheit der Dinge enthalten?! Diesen Fragen eines skeptischen Idealismus lässt sich auf doppelte Weise begegnen. Entweder nämlich - man begrëift die Ideen als die methodischen Grundbegriffe der Wissenschaften, als die „Hypothesen“, die den Gegenstand ursprünglich aus sich zur Erzeugung bringen. Das führt zu dem "methodischen" oder „kritischen “ Idealismus Kants, der als ${ }_{n}$ methodisch" bezeichnet werden kann, weil nach ihm die Ideen nichts anderes bedeuten, als die fundamentalen Methoden 
einer Gegenstandsbestimmung überbaupt and der "kritisch" heisst, weil damit als die Aufgabe der Philosophie eine kritische Ontersuchung der Grundbegriffe und -Sätze der Wissenschaften, der „Doktrinen", erkannt ist. Das ist der Standpunkt der transscendentalen Methode. Vom ${ }_{n}$ transscendentalen ${ }^{*}$ a priori aber unterscheidet Kant ein "metaphysisches a priori". Und damit kommen wir zur zweiten Lösungsmöglichkeit, die der historischen Aufeinanderfolge nach die frühere ist, zu dem Idealismus des „metaphysischen a priori" oder dem dogmatischen Idealismus, wie er von Descartes, Malebranche und Leibniz vertreten wird.

Es soll nun keineswegs behauptet werden, dass diese scharfe Unterscheidung von den aufgeführten Philosophen stets streng beobachtet worden wäre. Vielmehr steht Kant in seiner vorkritischen Zeit und auch noch in der ersten Auflage seiner Vernunftkritik zum Teil noch auf dem Boden des dogmatischen Idealismus, während sich andererseits bei Descartes, Malebranche und vorzüglich bei Leibniz mannigfache Ansätze zum methodischen Idealismus finden. Was den dognatischen Idealismus im Grunde vom kritischen unterscheidet, ist das Fehlen des Prinzips des Ursprungs. Er entdeckt die Erkenntnisse der Vernunft als allgemein gültig und streng notwendig, d. h. als a priori, aber er findet nicht die kritische Ergänzung zum Begriffe des a priori, und so scheint es doch wieder, als ob diese Ideen und die Vernunft, die sie einschliesst, etwas Gegebenes wären, als ob sie ein „Sein" für sich hätten. Diese Auflösung des "mundus intelligibilis", wie es noch in der vorkritischen Schrift Kants heisst, vollzieht erst radikal der Begriff des Transscendentalen, durch den das "Sein" in Tätigkeit, in das Setzen fundamentaler Beziehungen aufgelöst wird und an die Stelle des Problems der "Welt" rein das der Erkenntnis tritt. Malebranches Idealisinus ist nun in der Hauptsache zwar dogmatischer Natur, aber es zeigen sich immerhin, besonders bei den Problemen des Unendlichen und des Raumes, Ansätze zum methodischen Idealismus, wie ihn; in schärferer Weise allerdings, vor Kant nur Leibniz vertritt. Ehe wir zur Betrachtung dieser beiden Begriffe übergehen, sei zunächst einiges Allgemeine über die Art des Malebrancheschen Idealismus gesagt.

Der zweite Teil des dritten Buches der „Recherche de la Vérité $^{\text {"1) }}$ ) hat die Überschrift: „De l'Entendement pur". Die

1) Zitiert nach der Ausgabe von F. Bouillier (Paris, Garnier) in 2 Bänden. 
Ideen sind dadurch charakterisiert als die Ideen des reinen Verstandes. Damit werden sie in Gegensatz gesetzt zu den Verstellungen der Sinne und der Einbildungskraft. Was versteht man denn nun aber unter einer „Idee“? Diese Frage beantwortet Malebranche folgendermassen: „Daran ist wohl kein Zweifel, dass wir die ausser uns befindlichen Dinge nicht durch sich selbst apperzipieren; denn sehen wir gleich die Sonne, die Sterne u.s. w. ausser uns, so ist es doch nicht wahrscheinlich, dass die Seele aus dem Körper hinaustritt und sozusagen im Himmelsgewölbe spazieren geht, um dort alle diese Dinge zu betrachten. Sieht unser Geist also z. B. die Sonne, so ist sein unmittelbares Objekt nicht die Sonne, sondern etwas, das innig mit unserer Seele verbunden ist, und dies ist es, was ich als Idee bezeichne. Demnach verstehe ich unter diesem Worte "Idee“ hier nichts anderes, als was dem Geiste das unmittelbare oder das nächste Objekt ist, wenn er etwas apperzipiert."

Mit dem ersten der obigen Sätze ist der Sensualismus abgelehnt; davon kann keine Rede sein, dass wir die „Dinge“ durch sich selbst apperzipieren, sie müssen also, soll von ihnen eine Erkenntnis möglich sein, zuerst in Vorstellungen aufgelöst werden. Sofern diese Vorstellungen nun der Seele als "unmittelbare Objekte“ entgegenstehen, bezeichnet sie Malebranche als Ideen. Damit der Geist etwas apperzipiert, ist es unbedingt notwendig, dass die Idee dieser Sache ihm aktuell gegenwärtig ist, es ist aber keineswegs notwendig, dass es ausser ihm etwas dieser Idee Ähnliches giebt. Der Geist hat also nicht nur die Fähigkeit, sich die wirklich vorhandenen Gegenstände vorzustellen, sondern er erstreckt sich auch auf die, welche nicht sind und die vielleicht nie gewesen sind, d. h. auf die möglichen Gegenstände. „So hat man häufig im Geiste reelle Ideen von Dingen, die niemals gewesen sind, so z. B. von einem goldenen Berge. Die Menschen leben nun in dem Vorurteil, dass sie, sobald sie ein Objekt wahrnehmen, glauben, gewiss sein zu dürfen, dass dieses Objekt existiert, wenngleich es häufig vorkommt, dass sich aussen gar nichts befindet, ja, noch mehr, sie glauben, dass dieses Objekt genau so ist, wie sie es sehen, was niemals zutrifft.“ "Das ist," wie in ähnlichem $\mathrm{Zu}$ sammenhange Descartes in den Meditationen ausführt, „der vorzüglichste und hänfigste Irrtum ... dass ich urteile, die in mir vorhandenen Ideen seinen gewissen ausser mir befindlichen Dingen ähnlich oder entsprechend “, und um diesen Grundirrtum zu wider- 
legen, macht er ebenda seine berühmte Einteilnng der Ideen. Malebranche fahrt an der obigen Stelle (R. I. 344) fort: "Was aber die Idee betrifft, die notwendig existiert und die nicht anders sein kann, als man sie schaut (voit), ${ }^{1}$ ) so urteilt man gemeinhin ohne weiteres Nachdenken, dass sie nichts ist, wie wenn die Ideen nicht eine grosse Anzahl von Eigenschaften (propriétés) hätten; wie wenn die Idee eines Quadrates z. B. nicht recht verschieden wäre von der irgend einer Zahl und nicht ganz verschiedene Gegenstände vorstellte, was für das Nichts nicht gelten kann, da ja das Nichts gar keine Eigenschaft hat." Das

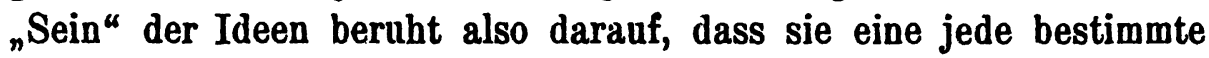
Gegenstände vorstellt, dass sie ideae tamquam rerum imagines nach dem Ausdrucke Descartes', und insofern nicht Nichts sind, d. h. eine bestimmte Anzahl von Eigenschaften aufweisen, die bei jeder Idee verschieden ist. "Il est donc indubitable que les idées ont une existence très réelle". Es wäre nun falsch, wollte man sich diese Existenz nach Art einer sinnlichen Gegebenheit vorstellen, nachdem Malebranche so klar und deutlich erklärt hat, dass ihr Sein bloss darin beruht, dass sie jede eine bestimmte Reihe von Eigenschaften haben, wodurch sie im Gegensatz zum Nichts als die Grundlagen der Prädikation dienen können. „Diese Realität der Ideen," so führen die Entretiens ${ }^{2}$ ) (S. 148) aus, „hängt nicht von meiner subjektiven Willkür ab; die Ideen haben eine ewige Wesenheit, sie entstehen und vergehen nicht in einem Augenblick. Dass man selbst vermöge seines persönlichen Bewusstseins den Ideen ihr Sein giebt, das ist nur insofern richtig, als die Aufmerksamkeit meines Geistes die ,Gelegenheitsursache ihrer Entstehung ist."

"Die Aufmerksamkeit ist," wie es in der Recherche (I, 337) heisst, "für die Objekte des Geistes das, was der feste Blick unserer Augen für die Objekte unserer Augen ist. Aber so wenig der Blick unserer Augen die sinnlichen Objekte schafft, so wenig schafft die Aufmerksamkeit die Ideen." Wie kann man überhaupt (Entretieus S. 14) an eine bestimmte Idee, z. B. einen Kreis denken

1) "voir" hat bei Malebranche stets die Bedeutung des geistigen" Sehens, Schauens, ist also fast gleichbedeutend mit connoître = erkennen; nur dass in voir mehr die Unmittelbarkeit des geistigen Erfassens liegt.

2) So zitiere ich der Kürze halber die „Entretieus sur la Métaphysique" (Ausgabe von J. Simon, Paris, Charpentier), die neben der "Re-1

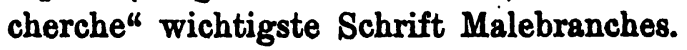


wollen, wenn man davon nicht schon irgend eine Idee hat, woraus soll man sie bilden und zustande bringen? Kann man etwas wollen, ohne zu erkenuen? Oder kann man etwas aus dem Nichts schaffen? Sicherlich kann man nicht an einen Kreis denken wollen, wenn man nicht schon seine oder zum mindesten die Idee der Ausdehnung hat." Die Aufmerksamkeit kann uns die Kreisidee höchstens gegenwärtig machen, vielleicht auch sie gestalten, nicht aber sie aus dem Nichts erzeugen. Und würde andererseits die Aufmerksamkeit den Kreis nicht nur entschwinden, sondern ihn gänzlich zunichte werden lassen, so wäre nicht einzusehen: erstens, wie man überhaupt wieder jemals wünschen könnte, den Kreis zu erzeugen; denn dieser Wunsch nach der Erkenntnis des Kreises setzt schon irgend eine Art des Wissens, sei sie auch noch so unbestimmt, voraus; zweitens, nach welchem Musterbilde (sur quel modèle) man ihn von neuem so ähnlich mit sich selbst machen würde (tout de nouveau si semblable à lui-même S. 15). Die Idee dient also als Musterbild, als „Modell“, nach dem, „auf welches schauend" - wie es in Platonischer Sprache lauten würde - der Geist immer von neuem Gegenstände, z. B. Kreise, die einander ähnlich sind, erzeugt. Oder, anders ausgedrückt, es bedeutet die Realität, das "Sein“ der Ideen - ihre begriffliche Bestimmtheit, die nichts den betreffenden ihr zukommenden Eigenschaften Widersprechendes duldet. Eine ähnliche Ausführung findet sich im ersten Bande der Sammlung der Streitschriften zwischen Malebranche und Antoine Arnauld: ${ }^{1}$ ) (S. 142). „Ohne Ideen vermag ich nichts klar zu erfassen... wenn ich nun z. B. an einen Kreis denke oder etwas durch eine Idee erkenne, so schaue ich es wie von mir getrennt" (je le voi comme séparé de moi). Bei der Erkenntnis durch die Ideen löst sich also das Etwas, das ich erkenne, gleichsam ab, es ist unabhängig von meiner persönlichen Willkür, meinem subjektiven Bewusstsein, es ist das diesem „Entgegenstehende ${ }^{\mu}$, „Widerstehende ${ }^{\mu}$ - das aber macht den erkenntnistheoretischen Begriff des "Gegenstandes" aus. Es ist dies ein ähnlicher Gedanke, wie wir ihn, nur in schärferer, kritischer Formulierung bei Kant finden, wenn' er in der Kritik der reinen Vernunft (Ausgabe Vorländer S. 708) sagt, dass der Gegenstand als dasjenige angesehen wird, was dawider ist, dass unsre Erkenntnisse nicht aufs Geratewohl oder beliebig, sondern a priori auf gewisse Weise bestimmt sind.

1) Ich zitiere sie kurz als "Recueil ${ }^{4}$.

Kantatudion Xrv. 
Es tritt nun, nachdem die. Ideen so charakterisiert sind, das Problem auf, in welcher Weise ich die Objekte vermöge ihrer Ideen erkennen kann. Malebranche erörtert in der Recherche (I, $311 \mathrm{ff}$.) sämtliche hier in Betracht kommenden Möglichkeiten; denn entweder müssen die Ideen, die wir von den Körpern haben, von diesen selbst kommen, oder unsre Seele hat die Fähigkeit, diese Ideen zu erzeugen, oder Gott hat diese Ideen mit ihr erzeugt, als er sie schuf - d. h. sie hat die Ideen als ein Besitztum in sich - oder er erzeugt sie jedesmal, wenn man an ein Objekt denkt, oder die Seele hat in sich alle die Vollkommenheiten, die sie in diesen Körpern sieht oder endlich, sie ist mit einem vollkommenen Wesen verbunden, das ganz allgemein alle Vollkommenheiten der geschaffenen Wesen einschliesst. Diese letzte Ausicht, dass die Seele mit dem vollkommensten Wesen, der universellen Wabrheit, vereinigt ist, ist die von Malebranche.

So gelangt er $\mathrm{zu}$ dem berühmten Hauptsatze seiner Philosophie: "Que nous voyons toutes choses en Diea", wie er seinen Gedanken ausdrückt. Er ist sich wohl der Schwierigkeiten und des Paradoxen dieser seiner Ansicht bewusst. "Ich habe hierüber" (sc. über die Frage, wie wir die Objekte erkennen) „eine Ansicht, die seltsam erscheint und mit der die Einbildung sich nicht leicht zufrieden giebt," schreibt er an Regis (S. 239) und wiederholt macht er in der Streitschrift gegen Arnauld (Recueil I. Bd.) darauf aufmerksam, dass die gewöhnliche, sinnliche Auffassung an dieser seiner Ansicht stets Anstoss nehmen werde und müsse. Das heisst, dass sich dieses Verhältnis zwischen Gott, der universellen Vernunft und unserer eingeschränkten Vernunft nicht irgendwie vorstellen lässt; es kann vielmehr nur durch den Verstand erfasst werden. Sehen wir nun zu, wie Malebranche im einzelnen

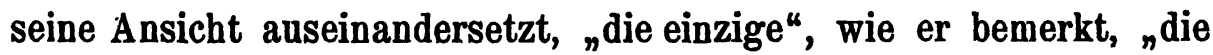
der Vernunft entspricht und die geeignetste, um die Abhängigkeit erkennen zu lassen, in der sich der Geist bei allen seinen Gedanken von Gott befindet" (S. 357).

Erstlich erscheint es notwendig, dass Gott in sich die Ideen all der Dinge hat, die er geschaffen. hat, da er sie sonst nicht hätte hervorbringen können, und dass er demnach alle Dinge schaut, indem er das in sich betrachtet, was damit in Beziehung steht. Ferner ist Gott durch seine Gegenwart recht enge mit unseren Seelen vereinigt, sodass man sagen kann, dass er der Ort der Geister ist, ebenso wie der Raum der Ort der Körper 
ist. Auch bei diesem vielfach missverstandenen Vergleiche muss man sich des oben Gesagten erinnern, dass man nämlich das Verbältnis von Gott und unserem Geiste sich nicht sinnlich vorstellen darf. Es soll dies nur heissen: so wie der Raum die Bedingung für die Existenz wie für die Beziehungen darstellt, in denen sich die Körper zu einander befinden, so ist Gott die Bedingung für das Dasein wie für die Beziehungen, die zwischen den einzelnen Geistern stattfinden. Wie der Raum also als einig und unendlich die Bedingung für die Einheit der gesamten Körperwelt, so ist auch Gott, der ebenfalls einige und unendliche, die Bedingung für die Einheit der unter geistigen Wesen geltenden Vernunftgesetze.

Ist das nun richtig, dass der Geist alles in Gott schaut, so darf man doch daraus nicht schliessen, dass er damit sein Wesen (essence) schaut. Also man darf nicht glauben, dass mit diesem "Schauen" sogleich eine vollkommene Erkenntnis gegeben wäre; denn was der Mensch schaut, ist recht unvollkommen (dië einzelne Vernunfterkenntnis) während Gott böchst vollkommen ist - als die universelle Wahrheit, die als die Gesamtheit aller Vernunfterkenntnisse einschliessend gedacht werden muss. Was man schaut, ist immer ein Einzelnes, ein Besonderes, etwa auch Teilbares, Endliches, deshalb bleibt aber Gott oder die Vernunft selbst doch allgemein, unteilbar und unendlich. Übrigens kann man sagen, dass man nicht sowohl die Ideen der Dinge schaut, erkennt, als die Dinge selbst, welche die Ideen repräsentieren, denn wenn man z. B. ein Quadrat schaut, so sagt man nicht, dass man die Idee dieses Quadrats schaut, die mit dem Geiste vereinigt ist, sondern nur das aussen befindliche Quadrat.

Der stärkste Grund aber für die Richtigkeit des Hauptsatzes ist die Art und Weise, wie der Geist alle Dinge apperzipiert. Wenden wir uns nämlich einem besonderen Gegenstande zu, so haben wir daneben stets die Idee aller Gegenstände, d. h. eines Alls der Realität, von dem die einzelnen Gegenstände nur Folgen sind. Dieses All von Realität kann aber unserem Geiste nur gegenwärtig sein, weil Gott ihm gegenwärtig ist, er, der in seiner Einfachheit alle Einzelwesen einschliesst. Die Idee des Alls der Realität, des „Seins“ schlechtweg, kommt mit der Gottes, oder, wie man es auch ausdrücken kann, mit der des Unendlichen genau überein. Es steht also fest, dass der Geist das Unendliche apperzipiert, wenngleich er es nicht umfasst (comprend). Die Idee Gottes oder die des Unendlichen haben, das besagt genau 
dasselbe: "Dien ou l'infini", heisst es einmal in den Entretiens (S. 28) and noch genauer an einer andern Stelle desselben Werkes: "Il (Gott) est toujours un et toujours infini ... le rrai Dieu c'est l'Être . . . Dieu c'est l'infini en tont sens" (S. 185).

Indessen - es bleibt bei der Durchführung der These, dass wir alle Dinge in Gott schauen, noch eine Schwierigkeit: Wie ist es nämlich zu verstehen, dass wir in ihm auch die materiellen und sinnlichen Dinge schauen? Nicht so, dass wir in Gott die Empfindungen haben, sondern nur, dass wir sie v on Gott haben, der auf uns einwirkt; denn Gott erkennt allerdings die sinnlichen Dinge, aber er nimmt sie nicht wahr. Wenn wir etwas Sinnliches apperzipieren, so enthält unsere Vorstellung zweierlei: Empfindung und reine Idee. Die Empfindung ist eine blosse Modifikation unserer Seele, d. h. unseres Bewusstseins, die Gott verursacht und verursachen kann, weil er in der Idee, die er von unserer Seele hat, bemerkt, dass sie derselben fähig ist. Die Idee dagegen, die sich mit der Empfindung vereinigt findet, ist in Gott und wir schauen sie, wie oben erklärt. Sind nun die Objekte gegenwärtig, so vereinigt Gott die Empfindung mit der Idee und wir schliessen auf Grund der Empfindung auf das tatsächliche Vorhandensein des Gegenstandes, von dem wir, was er ist, nur durch seine Idee wissen.

Man hat schon zu seinen Lebzeiten Malebranche wegen dieser Aufstellungen Neigungen zur Mystik vorgeworfen, aber er ist auf einen derartigen Vorwurf gefasst und weiss ihm zu begegnen.

„Dieses Prinzip ${ }^{\alpha}$ - so führt er in der „Recherche (II, 106) aus, „dass allein Gott uns aufkläre (éclaire) und zwar durch die Manifestation einer Vernunft oder einer Weisheit, die unwandelbar und notwendig ist", dieses Prinzip scheine inm so der Religion entsprechend, dass er sich verpflichtet glaube, es gegen alle Angriffe weiter aufrecht zu halten. "Lieber,“ so fährt er fort, „mag man mich einen Visionär nennen oder mich als Mystiker (illuminé) behandeln und über mich alle jene billigen Spottverse ergiessen, mit denen die bei den kleinen Geistern allzeit geschäftige Einbildungskraft sich ja stets Vernunftgründen entgegensetzt, dit sie nicht versteht oder gegen die sie sich nicht verteidigen kann, als dass ich zugebe, dass die Körper im Stande seien, mich aufzuklären, und dass ich nur mich oder andere Menschen zu befragen brauche, die vielleicht grosses Geräusch in meinen Ohren 
anrichten, aber sicherlich nicht das Licht der Vernunft meinem Geiste zu bringen vermögen.“. Alle Spötteleien können ihn also in seiner Grundansicht nicht wankend machen und inn nicht davon überzeugen, dass eine wahrhaft gegründete Belehrung über die Gesamtheit der Dinge aus den Körpern, dem eigenen persönlichen Bewusstsein oder dem anderer Menschen zu gewinnen ist. Er geht nun zu den Gründen über, die seine Ansicht weiter zu stützen vermögen. -

„Jedermann stimmt wohl mit der Ansicht überein, dass alle Menschen imstande sind, die Wahrheit zu erkennen und selbst die unaufgeklärtesten Philosophen geben zu, dass der Mensch an einer gewissen Vernunft teilhat, die sie nicht näher definieren. Deshalb definiert man inn als animal rationis particeps, da sie alle ein wenigstens verworrenes Bewusstsein davon haben, dass das wesentlich Unterscheidende des Menschen in der notwendigen Beziehung (le rapport nécessaire) besteht, in der or $\mathrm{zu}$ der ,raison universelle seht. Ich erkenne z. B., dass $2 \times 2=4$ ist, dass man die Menschen den Tieren vorziehen muss $u$. a., und ich bin sicher, dass dies jeder Mensch in der Welt ebensogut erkennen kann wie ich." Malebranche führt hier eine Stelle aus Augustins Konfessionen an (Buch 12, Kap. 25): „Si ambo videmus verum esse quod dicis et ambo videmus verum esse quod dico, ubi quaeso id videmus? Nec ego nilque in te, nec tu in me, sed ambo in ipsa quae supra mentes nostras est incommutabili veritate" und bemerkt daza, dass, da ich diese Wahrheiten doch weder in dem Geist der anderen entdecke, noch sie dieselben in dem meinigen, es also notwendig ist, dass es eine „universelle Vernunft" giebt, die mich belehrt, ebenso wie alle übrigen Intelligenzen. Denn wäre die Vernunft, die ich befrage, nicht dieselbe, die auch dem. Chinesen auf seine Fragen antwortet, so könnte ich nicht so sicher sein, wie ich es bin, dass die Chinesen dieselben Wahrheiteu erkennen (voir), die ich erkenne.

Wie kommt es, das ist das Problem, dass die Gesetzlichkeiten meiner Vernunft nicht subjektiv bleiben, sondern allgemeine nnd notwendige Geltung haben? Was garantiert mir für den objektiven Wert meiner Ideen? Diese Frage lässt sich auf dogmatischem, vorkritischem Wege gar nicht anders lösen, als Malebranche es tut: ich muss eine allgemeine Vernunft annehmen, an der meine Vernunft teil hat. Und diejenigen Ideen sind wahr, die ich entdecke, indem ich in mich selbst eingehe und 
mich dann mit der universellen Vernunft verbinde, vereinige. Diese garantiert für den objektiven Wert meiner Ideen, die ohne diese Beziehung auf die allgemeine Vernunft meine Perzeptionen, meine blossen Vorstellungen, Schöpfungen meines endlichen Geistes sein und bleiben würden. Malebranche bemerkt hierzn: Es ist demnach die Vernunft, die wir befragen, wenn wir in uns selbst eingehen eine universelle Vernunft. ${ }_{n} \mathrm{Je}$ dis quand nous rentrons dans nous-mêmes, car je ne parle pas ici de la raison que suit un homme passionné. Lorsqu'un homme préfère la vie de son cheval à celle de son cocher, il a ses raisons, mais ce sont des raisons qui dans le fond ne sont pas raisonnables, parce qu'elles ne sont pas conformes à la souveraine raison, ou à la raison universelle que tous les hommes consultent." Das ${ }_{n}$ rentrer

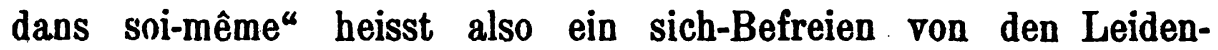
schaften, um rein die Vernunft walten zu lassen, sodass nur solche Gründe fortan gelten dürfen, die "raisonnables" sind.

In diesem Sinne ist es also doch das moi-même, das die Wahrheit in sich, d. h. indem es seine Vereinigung mit der raison universelle denkt, entdeckt. Es hat also das moi-même, das "Ich" bei Malebranche eine doppelte Bedeutung. In dem Ich $=$ Bewusstsein = Seele kann ich die Wahrheit nicht finden; meine Vorstellungen führen über sich selbst nicht hinaus, und hätte ich nichts als sie, so bätte ich nichts als ein "sentiment intérieur" von mir selbst, aber keine Erkenntnis (connoissance). Erkenntnis habe ich allein durch die Ideen, diese aber gehören meiner Vernunft an, setze ich also Ich $=$ Vernunft und denke ich diese als „raison universelle", so trete ich damit aus dem Kreise meiner bloss subjektiven Vorstellungen heraus. Denn dafür ist die universelle Vernunft die Garantie, dass gewissen Vorstellungen etwas Objektives, Unwandelbares, Ewiges entspricht, ein Etwas, das allen Menschen gemeinsam ist, und eben durch diesen Gedanken der Notwendigkeit und Allgemeinheit, der diese Vorstellungen begleitet, werden sie zu etwas mehr als bloss subjektiven Perzeptionen - zu Ideen.

Malebranche zeigt nun weiter, dass diese Vernunft unendlich ist. Denn man kann in ihr immer neue Gesetzlichkeiten entdecken, so z. B. immer neue Figuren. Der Geist erkennt also das Unendliche in dieser Vernunft, wenngleich er es nie. wird umfass en können; sie ist in der Hinsicht unerschöpflich, da sie stets etwas wird antworten können, gleichgültig, wonach man sie fragen mag. Und so ergiebt sich denn die enge Verwandtschaft der beiden 
Begriffe: „Vernunft" und „Gott". „S'il est vrai, que la raison à laquelle tous les hommes participent est universelle; s'il est vrai, qu'elle est infinie; s'il est vrai qu'elle est immuable et nécessaire; il est certain qu'elle n'est point différente de celle de Dieu même: car il n'y a que l'être universel et infini, qui renferme en soimême une raison universelle et infinie". Nehme ich also die universelle Vernunft einmal als gegeben, als vorhanden, als quasi daseiend an, so kann ich sie mit Gott identifizieren, denn: "Toutes les créatures sont des êtres particuliers: la raison universelle n'est donc point créée. Toutes les créatures ne sont point infinies: la raison infinie n'est donc point une créature. Mais la raison que nous consultons n'est pas seulement universelle et infinie, elle est encore nécessaire et indépendante, et nous la concevons en un sens plus indépendante que Dieu même. Car Dieu ne peut agir que selon cette raison: il dépend d'elle dans un sens: il faut qu'il la consulte et qu'il la suive." Sie hat also in einem Sinne eine noch grössere Notwendigkeit und Unabhängigkeit als Gott selbst; ihr Begriff liegt der Sache nach dem Gottes noch voraus. Ich kann dann auch, da Gott nichts anderes bedeutet, als Vernunft, beide gleichsetzen: „Or Dieu. ne consulte que lui-même: il ne dépend de rien. Cette raison n'est donc pas distinguée de lui-même: elle lui est donc coéternelle et consubstantielle." Erst so also, aus der Forderung einer Vernunft mit dem Charakter des Universellen, Notwendigen, Unabhängigen heraus, welche die Garantie bildet für die objektive Gültigkeit dessen, was die Einzelvernunft denkt, kommt Malebranche zu seinem Begriffe Gottes und: "alle Dinge in Gott scbauen" das heisst danach vichts anderes mehr, als sie in der universellen und notwendigen Vernunft schauen oder, kritisch gewendet, - was von Malebranche ja allerdings nur angedeutet werden kann, da er im Ganzen über den Dogmatismus eben nicht hinauskommt die Einzelgesetzlichkeit der Vernunft, das Besondere, aus der allgemeinen Gesetzlichkeit der Vernunft ableiten. -

Nach diesen grundlegenden Erörterungen, die notwendig waren, da über den Gehalt und Wert'der Malebrancheschen Ideenlehre keineswegs Klarheit und Übereinstimmung herrscht, gehe ich nun zu dem engeren Thema, der Idee des Unendlichen und dem Begriffe der intelligiblen Ausdehnung über.

Es gilt da zunächst festzustellen, dass der Begriff des Unendlichen sich bei Malebranche mit den mannigfachsten anderen 
Begriffen Verknupft; eine reine Herausarbeitung ist an keiner Stelle vorhanden. Andrerseits ist nicht zu leugnen, dass dieses stete Hineinspielen der Unendlichkeitsbetrachtung sich für seine Philosophie in mancher Beziehung als fruchtbar erweist. Es ist von vornherein als wahrscheinlich anzunehmen, dass die intensive Beschaftigung mit dem neuen Infinitesimal-Kalkül nicht ohne Bedeutung für die Entwicklung der philosophischen Gedanken Malebranches gewesen ist, zumal er einer der Ersten war, der die von Leibniz erfundene neue Rechnungsart verstand und anch sogleich in ihrer grundlegenden Bedeutung für die gesamte mathematische Naturwissenschaft begriff, wie es denn auch charakteristisch ist, dass gerade Malebranche der Lehrer desjenigen (de l'Hospital) gewesen ist, der das erste Lehrbuch über den neuen Kalkül geschrieben hat.

So lässt sich denn auch allein aus den wissenschaftlichen Ansichten Malebranches heraus der Begriff der étendue intelligible verstehen, der allen Gegnern und Erklärern bisher so grosse Schwierigkeit gemacht hat.

"Apperzipiert der Geist das Unendliche?" So lautet die Frage, mit der Malebranche für gewöhnlich in das Problem einführt (S. Entr. S. 18 ff.). Nicht in der Art, so muss die Antwort erfolgen, dass unser Geist einer unendlichen Ausdehnung gleichkäme. Immerhin aber erkennt (voit) der Geist doch, dass sein unmittelbares Objekt, die étendue intelligible, unendlich ist. Denn die Sache steht nicht so, dass er bloss nicht das Ende derselben sieht, sondern "il voit clairement qu'elle n'en a point". Die Jdee der intelligiblen Ausdehnung steht ihm in unerschöpflicher Weise zur Verfügung: „Il ne peut douter que cette idée ne soit inépuisable“. Diese intelligible Ausdehnung ist, wie Kant sagen würde, „eine unendliche gegebene Grösse“. „Qu'il en prenne de quoi se représenter le lieu de cent mille mondes, et à chaque instant encore cent mille fois davantage, jamais cette idée ne cessera de lui fournir tout ce qu'il faudra. L'esprit le voit et n'en peut douter." Und zwar entdeckt der Geist nicht erst, dass sie unendlich ist, sondern er erkennt das unmittelbar aus ihrem Begriff: „il la voit actuellement infinie" und weiss deshalb, dass or sie nie erschöpfen wird.

"Les géomètres sont les plus exacts de ceux qui se mèlent de raisonner: or tous conviennent qu'il n'y a point de fraction, qui, multipliée par elle-même, donne huit pour produit, quoiqu'en 
augmentant les termes de la fraction on puisse approcher a l'infiui de ce nombre. Tous conviennent que l'hyperbole et ses asymptotes et plusieurs autres semblables lignes, continuées à l'infini, s'approcheront toujours sans jamais se joindre, Pensez-rous qu'ils découvrent ces vérités en tâtonnant et qu'ils jugent de ce qu'ils ne voient point par quelque peu de chose qu'ils auraient découvert? ... Les vrais philosophes ne jugent précisément que de ce qu'ils voient. Et cependant ils ne craignent point d'assurer, sans jamais l'avoir éprouvé, que nulle partie de la diagonale d'un carré, fût-elle un million de fois plus petite que le plus petit grain de poussière, ne peut mesurer exactement et sans reste cette diagonale d'un carré et quelqu'un de ses côtés.“ Diese Beispiele zeigen, wie der Geist, ohne dass er jemals alle möglichen Fälle durchlaufen hat - was ganz unmöglich ist - dennoch eine unmittelbare Erkenntnis von den für das Unendlich-grosse wie für das Unendlich-kleine geltenden Beziehungen hat: „Tant il est vrai que l'esprit voit l'infini aussi bien dans le petit que dans le grand: non par la division on la multiplication réitérées de ses idées finies, qui ne pourraient jamais atteindre à l'infini mais par l'infinité même qu'il découvre dans ses idées et qui leur appartient, lesquelles lui apprennent tout d'un coup, d'une part qu'il n'y a point d'unité et de l'autre point de bornes dans l'étendue intelligible." Der Geist verfolgt also die Beziehungen bei dem Kleinen wie bei dem Grossen bis ins Unendliche und findet dabei in den Ideen selbst eine Unendlichkeit, d. h. eine Unendlichkeit von Beziehungen. Diese bleiben bestehen, ganz unabhängig von der absoluten Grōsse eines Objekts. Niemals, so klein das Quadrat auch sein mag, wird sich die Beziehung, das Verhältnis zwischen Seite und Diagonale ändern, und ebenso steht es mit der Hyperbel und ihren Asymptoten. Die Unendlichkeit, die der Geist in den Ideen findet, bedeutet also im Grunde, dass das Verfahren des Setzens von Verhältnissen schrankenlos zur Verfügung steht, und dass die Verhältnisse an keine bestimmte, endlich e Grösse gebunden sind. Es giebt keine absolute Einheit nach der Seite des Kleinen, keine absolute Schranke nach der Seite des Grossen, für welche die Verhältnissetzangen keine Geltung mehr hătten.

Das Unendliche hat eine wesentliche Eigenschaft: d'être en même temps un et toutes choses, d. h. Einheit und Allheit zu sein. Es ist „composé, pour ainsi dire, d'une infinité de por- 
fections et tellement simple qu'en lai chaque perfection renferme toutes les autres sans ancune distinction réelle ${ }^{a}$ (Fntretiens S. 31). Es enthält also einerseits eine Allheit von "perfections“, d. b. modern gesprochen, von Eigenschaften und ist andererseits doch so einfach, dass jede seiner Eigenschaften jede andere uumittelbar einschliesst, weil eben bei jedem Vergleichen der Eigenschaften z. B. der unendlichen möglichen Quadrate das Verhältnis zwischen Seite und Diagonale dasselbe bleibt. Nun kann ich von den unendlichen möglichen Dreiecken, von den unendlichen möglichen Quadraten usw. reden, es giebt also mehrere Unendliche und diese sind nicht gleich (35). „Tu dois savoir qu'il y a les mêmes rapports entre les finis qu'entre les finis et que tous les infinis ne sont égaux. ll y a des infinis doubles, triples, centuples les uns des autres; et quoique le plus petit des infinis soit infiniment plus grand qu'aucune grandeur finie, quelque grande qu'on la veuille imaginer, et qu'ainsi entre le fini et l'infini il ne puisse y avoir de rapport fini et que l'esprit humain puisse comprendre, néanmoins tu peux mesurer exactement les rapports de grandeur que les infinis ont entre eux; de même que tu peux souvent découvrir les rapports qui sont entre les nombres incommensurables, sans pouvoir jamais déterminer les rapports, que les nombres ont avec l'unité ${ }^{4}$. Also, dieselben Arten von Grössenbeziehungen sollen gelten $z$ wischen den Unendlichen wie zwischen den Endlichen: "les règles du fini réussissent dans l'infini“" wie Leibniz diesen Gedanken treffend zum Ausdrucke bringt. Ja, fährt Malebranche fort: "tu vois clairement que les infinis peuvent avoir entre eux des rapports finis - so sind im Differentialquotienten $d y$ und $d x$ als unendlich kleine Grössen gedacht und dennoch stellt ihr Verhältnis $d y$ : $d x$ eine endliche konstante Grösse dar - ils peuvent même avoir entre eux des rapports infinis; car l'esprit se représente des infinis infiniment plus grands les uns que les autres". Malebranche denkt hier wohl an die Grade des Unendlichen, d. h. daran, dass das Unendlich-kleine erster Ordnung z. B. „infinement plus grand" ist als das Unendlich-kleine zweiter Ordnung, das daher im Verhältnis zu ihm vernachlässigt werden kann, so wie bei der Vergleichung endlicher Grössen unendlich kleine Unterschiede (Differenzen) vernachlässigt werden. Betrachtungen ähnlicher Art finden sich auch in einer erst in den späteren Ausgaben hinzugefügten Stelle in der Recherche (36): „Jede endliche Grösse," heisst es hier, „verhält sich, 
verglichen mit dem Unendlichen oder geteilt durch das. Unendliche, zu dieser endlichen Grösse, wie diese selbe Grösse sich zu dem Unendlichen verhält. Das geht klar hervor aus demselben Grunde, der beweist, dass $1 / 1000$ sich verhält zu 1 wie $1 \mathrm{zu} 1000$, dass 2, 3, 4 Millionstel sich $\mathrm{zu} 2,3,4$ verhalten, wie $2,3,4 \mathrm{zu} \mathrm{2,}$ 3, 4 Millionen; denn wenn man auch ins Unendliche (infiniment) die Nullen vermehrt, so ist doch klar, dass die Proportion stets dieselbe bleibt. Es besagt das, dass eine endliche Grösse oder Realität gleich ist einer unendlich kleinen Realität des Unendlichen oder mit Bezug auf das Unendliche; ich sage mit Bezug auf das Unendliche (par rapport à l'infini); denn alles Grosse und Kleine ist gross und klein nur bezugsweise (par rapport)." Dies wird klarer noch durch das folgende: „Le produit de l'infini par l'infiniment petit est une grandeur finie et constante ... Cela est évident, et le fondement de la propriété des hyperboles entre les asymptotes, dont le produit des coupées croissantes à l'infini par les ordonnées diminuantes à l'infini est toujours égale à la même grandeur." Das heisst: Nehme ich bei der Hyperbel die Asymptoten als $x$ - und $y$-Achse, so wachsen mit dem Hyperbelast die Abscissen ins Unendliche, gleichzeitig nehmen die Ordinaten $a b$, bis sie schliesslich unendlich-klein werden, dennoch ist das Produkt $\mathbf{x} \cdot \mathbf{y}$ stets eine endliche, konstante Grösse, - das Endliche ergiebt sich also als das Produkt von etwas Unendlichgrossem mit etwas Unendlich-kleinem. -

Die angeführten Stellen zeigen, wie bei Malebranche der Begriff des Unendlichen, der bei Descartes noch mit metaphysischen Interessen aufs engste verknüpft erscheint (s. die 3. Meditation!), sich von diesen losgelöst hat und so durch die Bezugnahme anf die Mathematik, d. h. also anf die Wissenschaft, erst seine eigentliche Fruchtbarkeit erlangt.

Ich gehe nunmehr zu dem bereits erwähnten schwierigen Begriffe der intelligiblen Ausdehnung (étendue intelligible) über. Es mag zunächst erwähnt werden, dass dieser Begriff sich in den ersten drei Ausgaben der "Recherche ${ }^{*}$ nicht findet. Malebranche hat ihn erst später geprägt, um seine Lehre von dem Schauen in Gott $z \mathfrak{u}$ befestigen und $\mathbf{z u}$ klären. Über die Entstehung dieses Begriffes spricht er sich an einer Stelle des Recueil (I, 416 ff.) folgendermassen aus. Arnauld hatte spöttisch bemerkt, dass die ${ }_{n}$ intelligible $^{\mu}$ Ausdehnung für ihn etwas durchaus "inintelligible ${ }^{\text {“ }}$ (Unverständliches) sei, das ihm die Vorstellung eines materiellen 
Gottes erwecke, sodass or an dem Terminus geradezn Anstoss nehme. "Diesen anstoss-erregenden Terminus", erwidert ihm Malebranche, „habe ich vermieden, soweit ich konnte und zwar in der Furcht, die Delikatesse des einen oder anderen zu verletzen, sodass ich inn im ersten Bande der Recherche gar nicht gebrancht habe. Denn stets habe ich, soweit es mir möglich war, das zarte Gewissen der Schwachen geschont und die Böswilligkeit der Kritiker gefürchtet. Vor den,Eclaircissements ${ }^{(1)}$ ) habe ich stets ganz allgemein gesagt, dass wir in Gott seine Geschöpfe - die Dinge - schauen: „par ce qu'il y a en lui qui les représentoit".

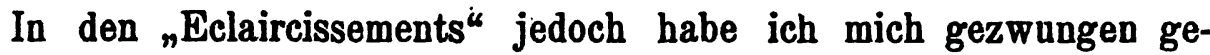
sehen, mich dieses so "skandalösen “ Ausdrucks der intelligibeln Ausdehnung zu bedienen, weil die Erfahrung mich gelehrt hatte, dass meine $\mathrm{zu}$ allgemein gehaltene Erklärungsweise einige wenig aufmerksame Personen in den groben Irrtum hatte verfallen lassen, als ob man die Sonne, ein Pferd, einen Menschen durch jede der Ideen schaute, die Gott in sich selbst hätte. Es ist dies indessen recht absurd; denn Gott schaut die Sonne unendlichmal grösser (nämlich in ihrer wahren Grösse), als die Menschen sie schauen. ${ }^{*}$ "Man muss," fährt Malebranche fort, „den.Autoren auch ein wenig Billigkeit entgegenbringen, besonders, wenn sie neue, abstrakte Wahrheiten vortragen. Es ist immer noch besser, die Einbildung, als die Vernunft zu verletzen und so dürfte diese meine Ansicht von der étendue intelligible wohl denen keine Schwierigkeit bereiten, die mit dem festgegründeten und uns erhebenden Satze wohl vertraut sind: „dass wir alle Dinge in Gott schauen."

Betrachtet man den Terṃinus: étendue intelligible, so könnte man auf den ersten Blick versucht sein, an eine Beeinflussung durch Aristoteles za glauben, der in seiner "Physik" von der

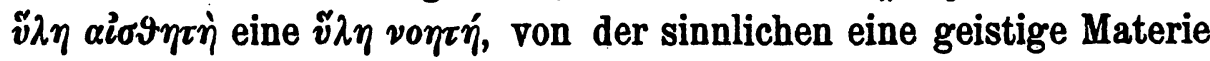
unterschied. Diese Unterscheidung ist dann von den Neuplatonikern, so von Plotin in der zweiten Enneade (4. Buch) aufgenommen und (wie mir scheint) in etwas veränderter Art durchgeführt worden. Bonitz in seinem Kommentar zur Metaphysik (ad $1036 \mathrm{aq})$ erklärt die $\tilde{v} \lambda \eta$ vo $\tau \dot{\eta}$ : „qualis est rerum mathematicarum materia, quae licet sit in rebus sensibilibus, tamen non eatenus in iis est, quatenus sunt sensibiles". Damit ist wenig gewonnen; denn wie nun diese intelligible Materie positiv zu

1) Gemeint sind die erst später hinzugefügten, ausführlichen „Er lauterungen" zu den Hauptlehren der "Recherche“. 
denken ist, das geht daraus ebensowenig hervor, wie aus einer anderen Stelle, wo sie erklärt wird als $\tilde{\eta} \tau \tilde{\omega} \nu \mu \alpha \vartheta \eta \mu \alpha \tau \iota x \tilde{\omega} \nu \tilde{v} \lambda \gamma$, als der der Mathematik zugrunde liegende ,Stoff'. Gerade dieser Aristotelische Gedanke, dass doch auch z. B. dem einzelnen Dreieck ein, wenngleich geistiger, Stoff zugrunde liegen muss, ist, wie wir noch genauer sehen werden, weit von dem entfernt, was Malebranche will. Immerhin ist möglich, dass Malebranche sich bei der Einführung seines Terminus der intelligiblen Materie erinnert hat, wie sie Plotin als die Materie der Ideen von der Materie der Sinnendinge unterscheidet.

Malebranche spricht sich einmal selbst darüber aus, weshalb er von einer étendue intelligible rede. In dem Briefwechsel mit de Mairan heisst es: (S. 307). „Er (es ist die Rede von Spinoza) verwechselt die geschaffene (d. h. die wirklich vorhandene) Ausdehnung, die nicht das unmittelbare Objekt des Geistes sein kann, mit der Idee von der Ausdehnung, die ich étendue intelligible nenne, weil sie allein es ist, die den. Geist afficiert (parce que c'est elle seule qui affecte l'esprit)“. Also sie heisst intelligibel, weil sie allein den Geist, die „intelligences“ affiziert. Die geschaffene Ausdehnung, d. h., da nach Malebranche Ausdehnung und Körper dasselbe besagt, der wirkliche, einzelne Körper kann den Geist nicht affizieren, kann auf ihn nicht unmittelbar einwirken. Eine solche unmittelbare „Einwirkung“ auf den Geist ist allein möglich durch die ideale, die intelligible Ausdehnung. Wir schauen eben, das ist eine Grundvoraussetzung bei Malebranche, die Körper nicht unmittelbar und in sich selbst (Vergl. z. B. Entretiens S. 326), sondern nur in ihren Ideen oder ihren „Archétypes“. Es hängen also keineswegs, wie die Sensualisten annehmen mögen, diese Ideen von den „êtres créés comme de leur cause exemplaire ${ }^{\alpha} a b$, pnisqu'elles sont au contraire les exemplaires des êtres créés". Die Ideen sind die Urbilder oder die Muster (exemplaires), von denen die „Ectypa“, die Einzeldinge abhängen. (An Regis 264 f.) So kann man denn die Körper schauen nur dadurch, dass der Geist die Idee der Ausdehnung sich gegenwärtig hält. „Il est donc certain qu'on ne voit les corps que dans l'étendue intelligible et générale" (An Regis S. 241). Wie ist nun die étendue intelligible zu charakterisieren? "Nichts“, heisst es in den Entretiens (S. 187) „ist klarer als die étendue intelligible. " ${ }^{\circ i e n}$ n'est plus intelligible que les iaees des corps. puisque c'est par elles que nous connaissons fort distinctement, non 
la nature de Dieu, mais la nature de la matière." Die Natur, d. h. die wesentlichen Eigenschaften der Materie, werden also durch sie erkannt. Das heisst aber nicht, dass sie "de même nature ist, wie die Materie. Sie ist vielmehr die Idee der Materie (Recueil I, 371). „Je n'ai jamais pensé que l'étendue fût plus grande dans un plus grand espace et plus petit dans un plus petit" (Recueil I, 354). Man muss eben scharf auseinanderhalten die Idee der Materie, des Körpers und die Materie, die Körper selbst. Diese sind „localement étendus" und nehmen so Raum ein, aber die Idee der Körper ist doch nicht selbst ein körperlich.(= ränmlich) Ausgedehntes, wenngleich sie die Bedingung für die Existenz solcher ausgedehnter Körper darstellt. Das bedeutet es, wenn die étendue intelligible als der Archetypus bezeichnet wird „du monde matériel que nous habitons et celui d'une infinité d'antres possibles". Sie ist das Urbild, dàs einer Unendlichkeit möglicher Welten zugrundeliegt (Entr. S. 26 f.). Sie ist die Idee oder der Archetypus der Körper (S. 29).

Das Verhältnis zwischen der Idee der Körper und diesen selbst wird dann noch klarer an einer anderen Stelle der Entretiens (S. 31): Die étendue intelligible repräsentiert die Körper; sie ist ihr Archetypus oder ihre Idee. Aber obgleich diese Ausdehnung - als Begriff, als Archetypus - keinen Ort einnimmt, sind die Körper selbst „étendus localement". So repräsentiert die intelligible Ausdehnung unendliche Räume, aber sie selbst erfüllt keinen. Wenn sie etwas erfüllt, so höchstens den Geist: „Et quoiqu'elle remplisse, pour ainsi dire, tous les esprits et se découvre à eux, il ne s'ensuit nullement que notre esprit soit spacieux". Er müsste ja eine unendliche Ausdehnung haben, um die unendlichen Räume zu schauen, wenn das Schauen nur durch eine lokale Vereinigung möglich wäre.

Die étendue intelligible ist also nicht selbst als ein Stoffliches, ein Dingliches zu denken, sondern als eine Vorstellung, ein Begriff, der notwendig "in uns", d. h. in unseram Geiste anzutreffen ist. Diese intelligible Ausdehnung ist nicht „faite " „elle est éternelle, nécessaire, infinie" (Brw. mit de Mairan S. 307). Die wirklichen, ausgedehnten Körper kann man sich als "faits“, d. h. entstanden denken, auch vergehen sie wieder und erleiden Veränderungen aller Art, aber alle diese Veränderungen sind $\mathbf{z u}$ beziehen auf die eine und unendliche intelligible Ausdehnung, die als relativ absoluter Bezugspunkt sich selbst nicht verändert, viel- 
mehr als „Archetypus" etwas von der Zeit Unabhängiges (ëternelle) und schliesslich notwendig ist. Malebranche begreift gar nicht, wie man hier eine Schwierigkeit finden kann; denn es ist doch klar, der Unterschied zwischen der Idee einer Sache und der Sache selbst, zwischen der geschaffenen Ausdehnung, die ich als materielle bezeichne, d. h. derjenigen, aus welcher sich die Welt zusammensetzt . . . und der Idee, die Gott davon hat und mit der er meinen Geist affiziert (S. 309). Die intelligible Ausdehnung ist also die Idee, mit der Gott mich affiziert. Nun hatten wir oben gesehen, dass sie notwendig ist. „Les idées que Dieu a sont les mêmes que les nôtres, quand elles sont nécessaires" ist aber ein Axiom, das Malebranche an derselben Stelle (S. 308) aufstellt. Meine Idee von der intelligiblen Ausdehnung ist also dieselbe wie die Gott hat.

Denke ich an diese Ausdehnung mit geschlossenen Augen, also unabhängig von den Sinneseindrücken, so stellt die Idee der Ausdehnung sie mir als unermesslich (immense) und gleichartig (partout la même) vor. Diese Ausdehnung denke ich mir also als unendlich und in sich gleichartig und ich nenne die Ausdehnung deshalb intelligibel, weil "diese Idee mich nicht durch die Sinne affiziert (S.309). Dieses nicht sinnliche Affizieren nennt Malebranche eiu Affizieren „d'une pure perception“. Öffne ich nun aber die Augen, so sage ich, dass es dieselbe Idee ist und keineswegs eine andere, die mich mit den sinnlichen Perzeptionen affiziert, die man rote, grüne, blaue Farben nennt, es wird alsdann diese selbe Idee „sensible“, während sie vorher „intelligible“ war, d. h. sie affiziert mich mit sinnlichen Perzeptionen.

Von dem von den Sinneseindrücken freigedachten Raum habe ich also eine reine Vorstellung. Dieser Begriff der ,reinen Vorstellung" erinnert, wie mir scheint, an Kant. Dieser definiert in der Kritik der reinen Vernunft (S. 68). „Ich nenne alle Vorstellungen rein, in denen nichts, was zur Empfindung gehört, angetroffen wird." Die Ähnlichkeit geht noch weiter, wenn Malebranche erklärt „j'appelle cette étendue, intelligible؛, parce que cette idée ne m'affecte point par mes sens " und Kant feststellt, dass Ausdehnung und Gestalt zur reinen Anschauung gebören und bemerkt, dass eine nicht empirische Ausdehnung eine solche sei, die auch ohne einen wirklichen Gegenstand der Sinne oder Empfindung (als eine blosse Form der Sinnlichkeit) im Gemüte stattfindet." Auch sind nach der übereinstimmenden Ansicht 
Kants und Malebranches die reine und die empirische Anschaung nicht getrennt, vielmehr, wie Malebranche sagt, ist es dieselbe Idee, die vorher "intelligibel" war, als noch von den Sinneseindrücken abstrahiert wurde, und jetzt dadurch ${ }_{n}$ sensibel $^{\mu}$ geworden ist, dass diese hinzugekommen sind.

Aber, so kőnnte man sagen, darin liegt doch der eigentliche Wert des Kantischen Begriffs vom Raume als reiner Anschaung, dass er in Zusammenhang gedacht wird mit der Wissenschaft der Geometrie, die er in ihrer "Möglichkeit" begründet. Wie steht es nun in dieser Beziehung mit Malebranches Begriff der étendue intelligible? Setzt Malebranche sie in Beziehung zur Wissenschaft? Allerdings, und zwar nicht nur einmal, sondern durchweg: „Arnauld ne scauroit deviner ce que j'ai voulu qu'on entendit par cette étendue intelligible infinie, dans laquelle je prétends que nous voyons toutes choses." Dann, meint Malebranche, hat er (Arnauld) eine Geometrie geschrieben "sans avoir d'idée de l'objet unique de cette science, car la Géométrie n'a point d'autre objet que l'étendue intelligible (Recueil I, 104). Das heisst doch klar: die Geometrie hat gar keinen anderen Gegenstand, als die intelligible Ausdehnung; diese ist demnach nichts anderes als der gleichartige, unendliche Raum, wie ihn die geometrischen Axiome voraussetzen und zur Bestimmung bringen.

Fast noch deutlicher heisst es an einer Stelle der Entretiens (S. 186): „Nous connaissons fort clairement l'étendue intelligible et si clairement que c'est en elle et par elle que les géomètres découvrent toutes leurs démonstrations." Die intelligible Ausdehnung dient also als die Voranssetzung, die notwendige Hypothesis. vermöge deren allein die Geometer alle ihre Sätze entdecken. Kommt das nicht dem Kantischen Gedanken der transscendentalen Deduktion wenigstens nahe, wenn Kant sagt, dass der Raum als ein "Prinzip" begriffen werden müsse, aus dem die Möglichkeit anderer synthetischer Erkenntnisse a priori eingesehen werden kann? (S. 73). Bei Kant heisst es: der Raum als reine Anschauung ist die Bedingung a priori der Möglichkeit der Geometrie; bei Malebranche: der Raum als étendue intelligible ist die einzige Bedingung, auf Grund deren die Geometer alle ihre Sätze entdecken.

Die sachliche Übereinstimmung scheint keines weiteren Beweises zu bedürfen. Es sei indessen noch eine Stelle aus den Entretiens angefïhrt, die eine grosse Ähnlichkeit in der Charakte- 
risierung des Raumes bei Malebranche und bei Kant zeigt. In den Entretiens heisst es S. 17: „Vous avez l'idée de l'espace ou de l'étendue, d'un espace qui n'a point de bornes. Vgl. Kant: „Der Raum wird als unendlich gegebene Grösse vorgestellt" (S. 72). "Cette idée est nécessaire" [Kant: der Raum ist eine notwendige Vorstellung (S. 71)] éternelle, immuable, commune à tous les esprits, aux hommes, aux anges, à Dieu même". Weiter heisst es dann: "Cette idée est ineffaçable de notre esprit ... elle lui est toujours présente". Kant: Man kann sich niemals eine Vorstellung davon machen, dass kein Raum sei, - das heisst doch wohl, dass man ihn nicht: ,effacer de l'esprit' kann - ob man sich gleich ganz wohl denken kann, dass keine Gegenstände darin angetroffen werden. Malebranche bezeichnete oben den Raum als eine notwendige und allen denkenden Wesen gemeinsame, d. h. allgemeine Idee; das heisst bei Kant: „der Raum ist eine Vorstellung a priori.“

„Vous ne pouvez vous en séparer ou la perdre entièrement de vue. Or c'est de cette vaste idée que se forme en nous non seulement l'idée du cercle et de toutes les figures intelligibles, mais aussi celle de toutes les figures sensibles que nous voyons en regardant le monde créé (die wirkliche Welt): tout cela selon les diverses applications des parties(!) intelligibles de cette étendue idéale, immatérielle, intelligible à notre esprit". Also - alle Figuren werden erkannt allein aus dieser "vaste idée“, die, wie es später (S. 19) heisst: „inépuisable“ ist. Und auch die Gestalten der Sinnendinge erkennen wir so; denn ihre Erkenntnis ist möglich allein durch eine bestimmte Anwendung der intelligiblen Figuren auf das Sinnliche.

Wir hatten oben gesagt, dass die intelligible und die materielle Ausdehnung nicht als getrennt zu denken sind. Das Verhältnis beider wird nun vortrefflich in einem Beispiel erläutert, das Malebranche im Recueil braucht (S. 151 des I. Bds). Bei der Perzeption, d. h. ganz allgemein: dem Erfassen der materiellen Objekte ist zweierlei vorhanden: sentiment confus und idée claire. So findet sich in der Perzeption, die ich von einer Marmorsäule habe 1. die Idee der Ausdehnung vor; diese ist klar und 2. die verworrene Empfindung der weissen Farbe, die sich darauf bezieht (qui s'y rapporte). Also die Empfindungen beziehen sich auf die Ausdehnung! Die Farbe selbst nämlich, das bedarf keines Beweises, ist nichts als eine Empfindung oder eine Modifikation der Seele. Angenominen nun, diese Säule werde ihrer Farbe 
beraubt, oder die Farbenempfindung, die sich darauf bezog, beziebe sich nicht mehr daranf - so werde ich sicherlich die Ausdehnung der Säule nicht mehr sehen; denn es ist sicher, dass man die Ausdehnung nur sieht durch die Perzeption oder Modifikation der Seele, die man als Farbe bezeichnet. Also dazn, dass ich diese besondere Ausdehnung wahrnchme, bedarf es freilich der Farben. Nun weiss ich aber doch, dass diese weisse Farbe der Marmorsäule nicht wesentlicb ist, daher werde ich mir trotzdem einen Begriff, eine Vorstellung von ihrer Ausdehnung - als dem ihr Wesentlichen - machen; wenngleich eben diese Ausdehnung sinnlich nicht festzustellen ist (j'y concevrai toujours son éteudue, quoiqu' invisible) und alsdann wird die Idee meines Geistes eine intelligible Säule sein. Dass ich die Säule als weiss sah, war bedingt durch meinen Organismus; jeder andere Beobachter wird eine andere, ihn besondere, niemals mit der meinen genau übereinstimmende Empfindung haben, aber die intelligible Säule, d. h. die hestimmt gestaltete Ausdehnung ist ein allen denkenden Menschen gemeinsames Objekt, "tausend Menschen könneu eine und dieselbe Säule sehen, „je la prens numériquement“; d. h. tausend verschiedene Beobachter beziehen ihre tausend verschiedenen Empfindungen auf eine und dieselbe ausgedehnte Säule. Diese intelligible Ausdehnung, auf welche die Farbe sich bezieht und durch welche sie sichtbar ist, ist nun keine blosse Modifikation meiner Seele mehr, sondern von ihr habe ich als dem aller Wahrnehmung Zugrundeliegenden und diese allererst ermöglichenden eine klare Idee. Die étendue intelligible ist also hier offenbar ähnlich gedacht wie bei Kant die allen Empfindungen „zum Grunde liegende Vorstellung“ des Raumes; denn damit die tausend Personen ihre Empfindungen auf etwas ausser ihnen Befindliches beziehen, muss die eine „intelligible Säule“ zum Grunde liegen.

Betrachten wir nun zum Schluss; wie Malebranche diesen seinen Begriff der étendue intelligible in den "Eclaircissements“ gegen die Angriffe der Gegner verteidigt und wie er ihn im genaueren durchführt. "Warum", so lautet der erste gewichtige Einwand, „soll die Idee der Ausdehnung nicht eine der Modifikationen der Seele sein“? (Recherche, Buch III, S. 127). Die Ausdehnung, erwidert Malebranche, kann kein solcher Modus der Seele sein, denn sie ist überhaupt kein Modus, sondern wahrhaft ein Sein (pas une manière d'être, c'est véritablement un être). 
Man kann sie allein denken, unabhängig von etwas anderem, vor allem aber unabhängig vom Geiste, es ist selbst unbegreiflich, dass die Ausdehnung ein Modus unseres Geistes sein könnte." Das ist sehr wohl zu verstehen; denn der Begriff der Ausdehnung ist fundamental verschieden von dem der Seele: wie die Ausdehnung als der Grundbegriff für die Erklärung alles Physischen, so dient das Bewusstsein als der Grundbegriff für die Erklärung alles Psychischen. Es geht aber nicht an, das Physische als einen Modus des Psychischen zu denken. „Die Einschränkung dieser Ausdehnung ruft eine Figur hervor, die Einschränkung des Geistes aber "keine geistige "Figur“, Gestalt. Da die Ausdehnung Teile hat, so unterliegt sie der Eigenschaft der Teilbarkeit, nicht so die Seele." "Diese Ausdehnung," so schliesst Malebranche, „ist also keine Modifikation des Geistes; da dieser nun nur seine Modifikationen in sich schaut, so schaut er sie nicht in sich. Wie könnte man auch in einer hesonderen espèce - wie unser Geist eine ist - „toutes les espèces des êtres" schauen und in einem besonderen und endlichen Wesen die allgemeine Idee, z. B. eines Dreiecks oder unendlich viele Dreiecke! Denn das ist unzweifelhaft, dass die Seele ein Dreieck im allgemeinen apperzipiert, d. h. eine allgemeine Idee des Dreiecks hat, wenngleich es widersprechend ist, dass sie eine allgemeine Modifikation hat; Modifikationen können eben ihrem Begriffe nach nur besonders sein. Fügt also die Seele zu einer Figur bestimmte Farben-Empfindungen hinzu, so machen diese damit die Figur zu etwas Besonderem, Einzelnem.

Ist die Ausdehnung also kein Modus des Bewusstseins, was ist sie denn? Malebranche bedient sich hier des Descartischen Prinzips, dass man das aussagen (assurer) kann, wovon man einen klaren Begriff hat (ce que l'on conçoit clairement). Nun hat man einen klaren Begriff davon, dass die Ausdehnung, die man schaut, etwas von uns Verschiedenes ist. Also kann man aussagen, dass sie keine Modifikation ist - Modifikationen sind eben etwas von uns nicht Verschiedenes - und dass sie tatsächlich etwas von uns Verschiedenes ist. Man muss nämlich bedenken, dass die Sonne, die man schaut (der Bewusstseinsinhalt, l'objet immédiat de notre pensée) etwas anderes ist, als die Sonne, die man sieht (d. h. unmittelbar empfindet).

Die Sonne, wie alles Materielle, ist ja, wie früher gezeigt, nicht durch sich selbst "visible", sondern allein durch die klare 
Idee ihrer selbst. Die geschante Sonne als Bewusstseinsinhalt ist, wenngleich mit meinem Bervusstsein unmittelbar vereinigt, etwas von meinem Bewusstsein, von "mir" Verschiedenes. „Donc on parle contre sa lumière et contre sa conscience, lorsqu'on dit que l'âme voit dans ses propres mudifications tous les corps qui l'environnent".

Alle unsere Empfindungen und Leidenschaften sind Modifikationen unserer Seele. Aber wir haben von ihnen keine klare Erkenntnis. Wir sind nicht imstande, sie mit einander zu vergleichen. Oder können wir etwa die exakten Beziehungen entdecken, die zwischen rot und grün oder selbșt zwisccen grün und grün vorhanden sind? Ganz anders steht es mit den eine bestimmte Einschränkung dèr Ausdehnung darstellenden Figuren! Deren Beziehungen unter einander lassen sich exakt feststellen, so z. B. dass das Quadrat über der Diagonale das Doppelte des Seitenquadrats ist.

Welcher Vergleich zwischen diesen intelligiblen Figuren, die recht klare Ideen, und den Modifikationen unserer Seele, die nichts als verworrene Empfindungen sind: es zeigt sich hier, dass die Seele apperzipieren kann, auch wenn das Betreffende nicht ihre Modifikation ist. Könnten wir die Figuren nur in uns selbst schauen, so würden sie „inintelligibles“ sein, da wir uns selbst „inintelligibles" sind. "Nous ne sommes que ténèbres à nousmêmes; il faut que nous nous regardions hors de nous pour nous voir." Nur in Gott, in der von unserem subjektiven Bewusstsein unabhängigen Vernunft, die wir insofern als „hors de nous" bezeichnen können, schauen wir alle Dinge. Und so ist denn auch die Idee der Ausdehnung, sofern wir sie in Gott und dadurch, dass wir sie in ihm schauen "très-claire".

"Indessen,“ so erhebt sich ein zweiter Einwand, „Gott ist in sich unwandelbar, wie kann ich also etwas so Wandelbares, wie etwa eine Figur, wahrnehmen?" - „Gott," antwortet Malebranche, "schliesst in sich eine unendliche intelligible Ausdehnung ein, da er doch die Ausdehnung erkennen muss, um sie schaffen zu können. Kann nun der Geist einen Teil dieser Ausdehnung apperzipieren, so kann er sicherlich in Gott alle Figuren apperzipieren; denn jede endliche intelligible Ausdehnung ist notwendig eine intelligible Figur, da die Figur nichts als die Begrenzung der Ausdehnung ist. Diese Figur mit intelligibler und allgemeiner, $d, h$. allgemein zur Verfügung stehender Ausdehnung 
wird dann sensibel und besonders, d. h. diese bestimmte, einzelne sinnliche Figur durch die Farbe oder irgend eine andere sinnliche Quälität, die die Seele damit verknüpft." Das heisst, indem auf die bloss geistige, allgemein zur Verfügung stehende Figur, z. B. des Kreises, die also eine reine Form bedeutet, eine Reihe von Empfindungen bezogen wird, entsteht diese hier und jetzt gegebene Figur. In Gott, der höchsten Vernunft, braucht es also keine sinnlichen Körper zu geben, da das, was die Figuren zu sinnlichen macht, Zutat unseres empfindenden Bewusstseins ist.

Es folgt nun eine schwierige, aber, wie mir scheint, tiefe Betrachtung, bei der Malebranche in der letzten Auflage der „Recherche“ bemerkenswerte Änderungen angebracht hat. Denkt man sich, dass eine Figur von intelligibler Ausdehnung, die durch die Farbe sinnlich gemacht worden ist, also etwa das gezeichnete Dreieck, successiv von den verschiedenen Teilen dieser unendlichen Ausdehnung aufgenommen wird, oder aber es drehe sich eine Figur von intelligibler Ausdehnung um ihr Zentrum oder nähere sich successiv einem anderen Zentrum, so bemerkt man (on apperçoit) die Bewegung einer sensibeln (materiellen) oder intelligiblen (mathematischen, also rein idealen) Figur, ohne dass in der intelligiblen Ausdehnung selbst aktuelle Bewegung vorhanden wäre. Es ist also die intelligible Ausdehnung unbeweglich „même intelligiblement". Statt dieses letzten Satzes haben die früheren Auflagen: „Peut-être que l'étendue intelligible est immobile en tout sens, même intelligiblement." Das "Vielleicht" ist in der letsten Ausgabe (1712) gefallen: die intelligible Ausdehnung ist in jeder Beziehung unbeweglich, sie muss es sein, weil sie ja die Bedingung enthält, auf Grund deren allein sich alle Bewegung der sinnlichen wie intelligiblen Figuren, d. h. alle Veränderung überhaupt zur Bestimmung bringen lässt. Also der Raum selbst ist als in sich unbeweglich $\mathrm{zu}$ denken; dennoch lässt sich alle Bewegung nur aus den in ihm gleichsam enthaltenen Beziehungen verstehen: "Wenngleich man aber voraussetzt, dass die intelligibeln Teile der Idee der Ausdehnung stets unter einander dieselbe Beziehung intelligibler Entfernung bewahren und dio intelligible Ausdehnung so selbst „intelligiblement“ unbeweglich ist, so wird man dennoch, wenn man sich eine bestimmte geschaffene Ausdehnung denkt (z. B. das gezeichnete Dreieck $\triangle$ ), die einem bestimmten Teile dieser Ausdehnung als ihrer Idee entspricht (correspond), eben durch die Idee des Raumes (espace), 
wenngleich sie "intelligiblement" unbeweglich ist, entdecken kounen, dass die Teile dieser geschaffenen Ausdehnung beweglich sind. Denn wenngleich die Idee des Raumes als "intelligiblement" unbeweglich vorausgesetzt wird, so stellt sie dennoch notwendig alle Arten von Entfernungsbeziehungen vor (représentant toutes sortes de rapports de distance) und macht es so begreiflich, dass die Teile eines Körpers unter einander ihre Lage verändern können." Also, um es knapp zusammenzufassen: der einige, unendliche Raum stellt, obwohl in sich unbeweglich, dennoch alle Arten von Entfernungs-Beziehungen vor und macht so die Lageveränderungen der Körper unter einander erst begreiflich und letztlich bestimmbar. Der Raum ist, wie Kant einmal (Kritik der reinen Vernunft S. 93) sagt, eine blosse "Verhältnisvorstellung“, und, wie es an einer anderen Stelle heisst: „Alles, was in unserer Erkenntnis zur Anschauung gehört," enthält "nichts als blosse Verhältnisse, der Örter in einer Anschauung (Ausdehnung), Veränderung der Örter (Bewegung) und Gesetze, nach denen diese Veränderung bestimmt wird (bewegende Kräfte). " "Wenngleich wir übrigens, " fährt Malebranche an der zitierten Stelle fort, "die Körper nicht in sich selbst schauen, sondern nur durch die intelligible Ausdehnung - mag diese nun „intelligible-

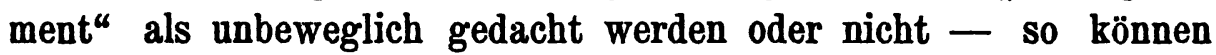
wir durch sie aktuell Körper in Bewegung sehen oder vorstellen, da sie uns als beweglich erscheint (parce qu'elle nous paraît mobile) auf Grund nämlich der Farbenempfindung oder der verworrenen Erinnerungsvorstellung, die wir successiv mit verschiedenen Teilen der intelligiblen Äusdehnung verknüpfen, die uns als Idee dient, wenn wir die Bewegung eines Körpers sehen oder vorstellen. Sehen wir also einen Körper sich bewegen, so verknüpfen wir unsere wechselnden Empfindungen mit verschiedenen Teilen des als selbst unbeweglich zugrundeliegend gedachten Raumes und allein durch diese Verknüpfung vermögen wir die Bewegung des Körpers eigentlich festzustellen, über welche uns die Empfindungen an und für sich, losgelöst von dieser Beziehung, nicht belehren würden. Die Übereinstimmung in den obigen Ausführungen Malebranches mit Plato ist unverkennbar, der die Geometrie als die Wissenschaft des á $\varepsilon i$ ǒv bezeichnet (Staat 527 B).

"Man kann also auf diese Weise, meint Malebranche, verstehen, inwiefern man die gedachte Sonne bald klein, bald gross 
schauen kann, wenngleich sie mit Bezug auf Gott, d. h. in Wahrheit, stets ein- und dieselbe ist. Denn es genügt hierfür, dass wir bald einen grösseren Teil der intelligiblen Ausdehnung schauen und bald einen kleinen. Denn da alle Teile der intelligiblen Ausdehnuug von derselben Natur sind, so können sie alle jeden beliebigen Körper vorstellen." Diese Stelle erscheint bei Malebranche nicht recht klar; vielleicht meint er aber gar nichts anderes, als was Descartes mit seinem bekannten Beispiel von den beiden Sonnenideen sagen will. Es giebt zwei Ideen von der Sonne (Malebranche sagt: mehrere „soleils intelligibles“) und zwar kommt der Sonne (Malebranche: so wie sie Gott schaut) die astronomische näher; für alle "Sonnenideen" aber steht die étendue intelligible als die Grundlage zur Verfügung, da alle ibre Teile „de même nature" sind und so jeden beliebigen Körper vorstellen können. -

Es gilt hier einzuhalten: der enge Rahmen eines Aufsatzes erlaubt es nicht, zu zeigen, obgleich sich das an sich wohl verlohnen würde, wie sich in Malebranches Lehre von der Wahrheit und der wissenschaftlichên Methodik die so gewonnenen Begriffe als fruchtbar erweisen. Indem diese Darlegungen einer andern Gelegenheit vorbehalten bleiben mögen, hoffe ich doch in obigem bereits klar gezeigt $\mathrm{zu}$ haben, dass Malebranche durchaus nicht in dem Grade, als man für gewöhnlich annimmt, von Descartes abhängig ist, dass er vielmehr in seiner Ideenlehre einige recht charakteristische, originale und für die Fortentwicklung der Philosophie bedeutungsvolle Züge aufweist. 